\title{
CLAVE DE IDENTIFICACION PARA GASTEROPODOS PULMONADOS DULCEACUICULAS DE IMPORTANCIA MEDICA EN EL PERU
}

\author{
RONALD VIVAR G. (*, **), \\ PEDRO HUAMAN M. $\left(*,{ }^{* *}\right)$ \\ HERNANI LARREA C. (**).
}

\author{
(*) - Laboratorio de Invertebrados, Facultad de Ciencias Biológicas, Universidad Ricardo Palma. \\ (**) - Laboratorio de Fauna Dulceacuícola, Facultad de Ciencias Biológicas, Universidad Nacional Mayor de San Marcos.
}

\begin{abstract}
RESUMEN
Se presenta una clave de identificación para gasterópodos pulmonados dulceacuícolas, considerando aspectos anatomicos del sistema reproductor.
\end{abstract}

\section{SUMMARY}

A key for indentification of freshwater pulmonate gastropods based on the anatomical characteristics of the reproductive system are presented.

\section{INTRODUCCION}

La fauna malacologica, dulceacuícola en nuestro país es altamente diversificada en forma y tamaño, presentando una distribución geográfica muy amplia en ambientes acuáticos en nuestro territorio (Huamán et. al. 1991).

La clasificación de las diferentes familias de gasterópodos durante varias décadas se fundamentó en detalles de la conchilla (Arrarte et. al. 1975); actualmente caracteres anatómicos se consideran por ser estables y diversificados en otros grupos. (Malek, 1985); son de importancia para relacionarlos filogenéticamente.

La presente comunicación tiene como finalidad presentar una clave de identificación con detalles anatómicos que facilite el reconocimiento de los grupos más comunes.

\section{MATERIALES Y METODOS}

El material revisado fue colectado durante 1986 y 1993, en numerosas localidades de las diferentes regiones del país. Los procedimientos de colecta, fijación, preservación y microdisección han sido referidos en trabajos anteriores (Larrea et. al.; 1993; Vivar et. al.; 1993).

Esta información constituye parte de los estudios realizados sobre la fauna malacológica peruana; las muestras revisadas constituyen parte del material de las colecciones de moluscos de agua dulce de los laboratorios de Fauna Dulceacuícola (UNMSM)y de Invertebrados (URP) y delDepartamento de Malacología y Carcinología del Museo de Historia Natural (UNMSM).

\section{RESULTADOS}

Clave de identificación para gasterópodos pulmonados dulceacuícolas del Perú.

A. Conchilla con opérculo Familias Ampullaridae, Hydrobiidae, Thiaridae.

B. Conchilla sin opérculo $1,2,3,4$

1. Conchilla espiralada dextrógira FamiliaLymnaeidae

1.a Próstata ovoide, con hendidura profunda Género Fossaria

1.b Próstata rudimentaria, sin hendidura Género Pseudosuccinea.

2. Conchilla espiralada levógira Familia Physidae

2.a Prepucio con glándula dorsal apical Género Physa

3. Conchilla planiespiral

Familia Planorbidae

3.a Prepucio y pene simple, sin órgano prepucial ni flagelos

Género Biomphalaria

3.b Prepucio y pene unidos por la glándula prepucial, sin flagelos

Género Helisoma

3.c Prepucio y pene simple, sin órgano prepucial, con flagelos Género Drepanotrema

4. Conchilla pateliforme .......Familia Ancylidae

4.a Organo copulatorio con flagelo grueso, apical e invaginado Género Ancylus 


\section{COMENTARIOS}

Los gasterópodos dulceacuícolas destacan desde el punto de vista médico por su intervención en los ciclos biológicos de diferentes tremátodos (Fasciola hepatica, Schistosoma mansoni y Echinostoma spp). Con excepción de los Ancylidae, algunas especies de las familias Physidae, Lymnaeidae y Planorbidae son reconocidas como hospederos intermediarios de los parásitos citados; además, esta última tiene representantes que juegan un rol importante en el control biologico.
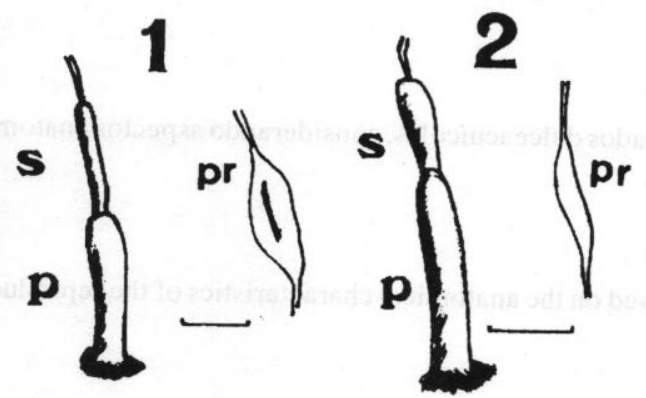

Fig. 1. Complejo penial y próstata de $\boldsymbol{F}$. viatrix. Fig. 2. Complejo penial y próstata de $P$. columella.

Dentro de los Lymnaeidae las diferencias en el sistema reproductor se pueden evidenciar en las Figs. 12. Fossaria viatrix tiene una próstata ovoide, ligeramente alargada con una hendidura profunda; Pseudosuccinea columella tiene una próstata rudimentaria, no distinguible en su parte anterior con el conducto espermático y en su parte posterior con el vaso deferente; el órgano copulativo en $F$. viatrix tiene una relación saco penial/prepucio de $2 / 3$, mientras que en $P$. columella la relación es menor de $1 / 2$.

La familia Physidae (poco estudiada en nuestro territorio) representada por dos especies del género $P$ hysa (venustula, peruviana), puede ser fácilmente reconocida porque el órgano copulatorio presenta una glándula prepucial en la porción dorsal y apical del prepucio; la próstata es digitiforme con pocos divertículos (ver Figs. 3-4); desde el punto de vista

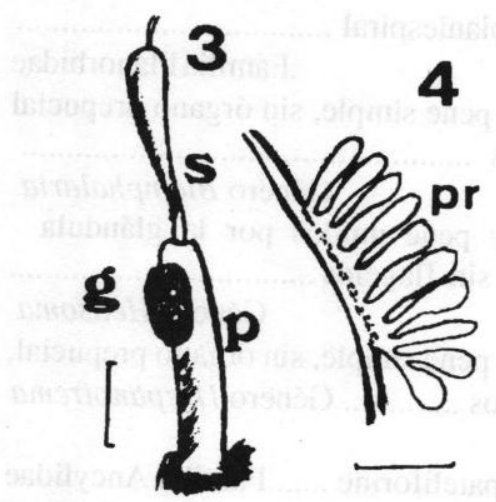

Fig. 3. Complejo penial de $P$. venustula. Fig. 4. Próstata de $P$. venustula. conquiológico $P$. peruviana es de mayor dimensión que P. venustula. de $\quad$ los Planorbidae, el $\mathrm{g}$ é n e r o Biomphalaria es reconocido por presentar un organo

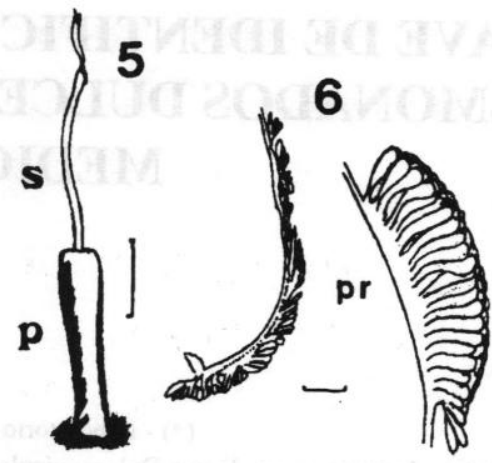

Fig. 5.Complejo penial de Biomphalaria.

Fig. 6. Próstata de B. tenagophila y de B. peregrina.

copulatorio simple (saco penial y prepucio); la próstata de las especies transmisoras de esquistosomiasis (Ver Figs. 5-6) es arborescente (B. tenagophila y B. glabrata) o digitiforme (B. peregrina). El género Drepanotrema presenta en el ápice de su saco penial flagelos glandulares delgados (Ver Figs. 7-8); su próstata puede ser digitiforme (D. kermatoides) o irregularmente sacular (D. cimex). El género Helisoma presenta un complejo penial abultado y un conducto prepucial que une al saco penial y al prepucio (Ver Figs. 9-10); su próstata es digitiforme. Las especies peruanas de Helisoma se diferencias por su órgano prepucial en forma de «copa» en trivolvis, de "pie» en duryi y ovoide vertical en peruvianum.

Los Ancylidae no han sido debidamente estudiados, el género Ancylus se reconoce porque su órgano copulatorio masculino presenta un flagelo apical grueso invaginado junto con la porción distal del prepucio (Ver. Fig. 11).

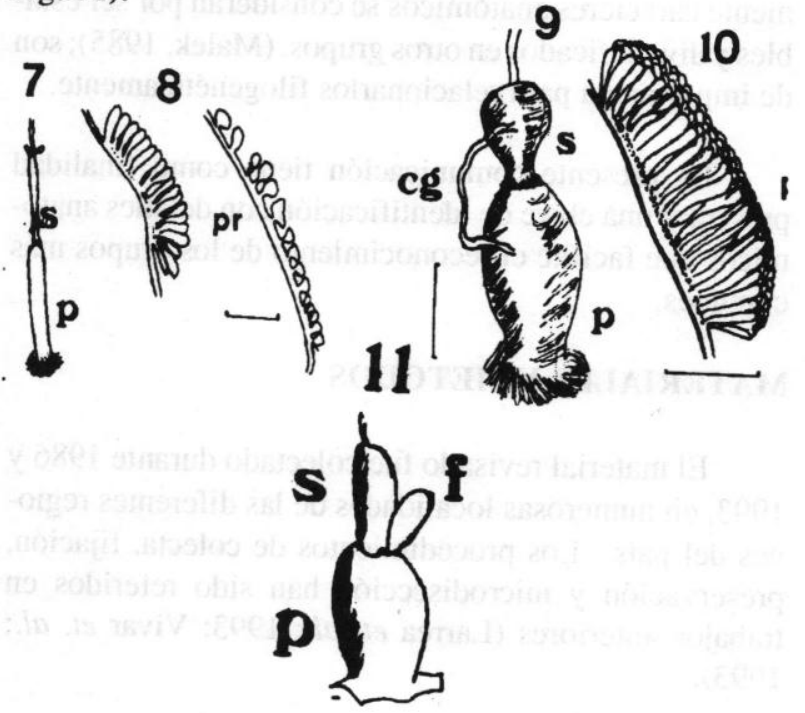

Fig. 7. Complejo penial de Drepanotrema.

Fig. 8. Próstata de D. kermatoides y D. cimex..

Fig. 9. Complejo penial de Helisoma.

Fig. 10. Próstata de Helisoma.

Fig. 11. Complejo penial de Ancylus.

Abreviaturas: $\mathbf{c} . g .=$ conducto de la glándula prepucial; $f=$ flagelos apicales; $\mathrm{g}=$ glándula prepucial; $\mathrm{p}=$ prepucio; $\mathrm{pr}=$ próstata; $\mathbf{s}=$ saco penial. (escala: $1 \mathrm{~mm}$ ). 
Consideramos que el reconocimiento de los géneros de gasterópodos dulceacuícolas, destacando los de importancia médica, tiene vital importancia en aspectos epidemiologicos. La necesidad de estudiar a las familias Physidae y Ancylidae considerando otros aspectos anatómicos sería de vital importancia para dilucidar algunas dudas en la sistemática de gasterópodos de agua dulce.

\section{AGRADECIMIENTOS}

Al Fondo de Desarrollo Universitario (FEDU) por el apoyo económico al Proyecto $\mathrm{N}^{\circ} 4100127 / \mathrm{ICBAR} /$ UNSNM.

A los doctores Emile Malek, W.L. Paraense y Gloria Perera por habernos proporcionado parte del material bibliográfico. A los Blgos. Liliám Pachas L. y Maria Luisa OviedoC.; por su asistencia técnica. A los señores Hugo López, Miguel Neira y Jaime Quesada por su eficiente colaboracion.

\section{REFERENCIAS BIBLIOGRAFICAS}

ARRARTE, J., M. BELLIDO y P. AGUILAR. 1975. Moluscos dulceacuícolas del Valle del Rímac, Lima, Peru. Anales Científicos U.N.A., 13: 14-21.

HUAMAN P., R. VIVAR, L. PACHAS, H. LARREA Y M.L. OVIEDO. 1991. Lista preliminar de gasterópodos dulceacuícolas y su importancia médica. Boletín de Lima, 77: 63-66.

LARREA, H., R. VIVAR, P. HUAMAN, L PACHAS Y M.L. OVIEDO. 1993. Clave para la identificación de las especies de la familia Lymnaeidae, vectores de la fasciolasis en el Perú. Boletín de Lima, 88: 3-8

MALEK, E. 1985. Snail hosts of schistosomiasis and other snail-transmitted diseases in Tropical America: A manual. World Health Organization, Scientific Publication $\mathrm{N}^{\circ} 478$ Washington. $325 \mathrm{pp}$.

VIVAR, R., L. PACHAS, P. HUAMAN, H. LARREA Y M.L. OVIEDO. 1993. Clave de identificación para los planórbidos peruanos de importancia médica y su distribución geográfica. I Parte. Revista Peruana de Medicina Tropical, UNMSM: 7: 89-98. 